\title{
Landau-Zener-Stückelberg-Majorana interference in a 3D transmon driven by a chirped microwave
}

Ming Gong, Yu Zhou, Dong Lan, Yunyi Fan, Jiazheng Pan, Haifeng Yu, Jian Chen, Guozhu Sun, Yang Yu, Siyuan Han, and Peiheng Wu

Citation: Appl. Phys. Lett. 108, 112602 (2016);

View online: https://doi.org/10.1063/1.4944327

View Table of Contents: http://aip.scitation.org/toc/apl/108/11

Published by the American Institute of Physics

\section{Articles you may be interested in}

Demonstration of superconducting micromachined cavities

Applied Physics Letters 107, 192603 (2015); 10.1063/1.4935541

Fast reset and suppressing spontaneous emission of a superconducting qubit

Applied Physics Letters 96, 203110 (2010); 10.1063/1.3435463

Population inversion induced by Landau-Zener transition in a strongly driven rf superconducting quantum interference device

Applied Physics Letters 94, 102502 (2009); 10.1063/1.3093823

Coplanar waveguide resonators for circuit quantum electrodynamics

Journal of Applied Physics 104, 113904 (2008); 10.1063/1.3010859

Electrically tunable superconducting terahertz metamaterial with low insertion loss and high switchable ratios Applied Physics Letters 109, 022601 (2016); 10.1063/1.4955454

Suspending superconducting qubits by silicon micromachining

Applied Physics Letters 109, 112601 (2016); 10.1063/1.4962327

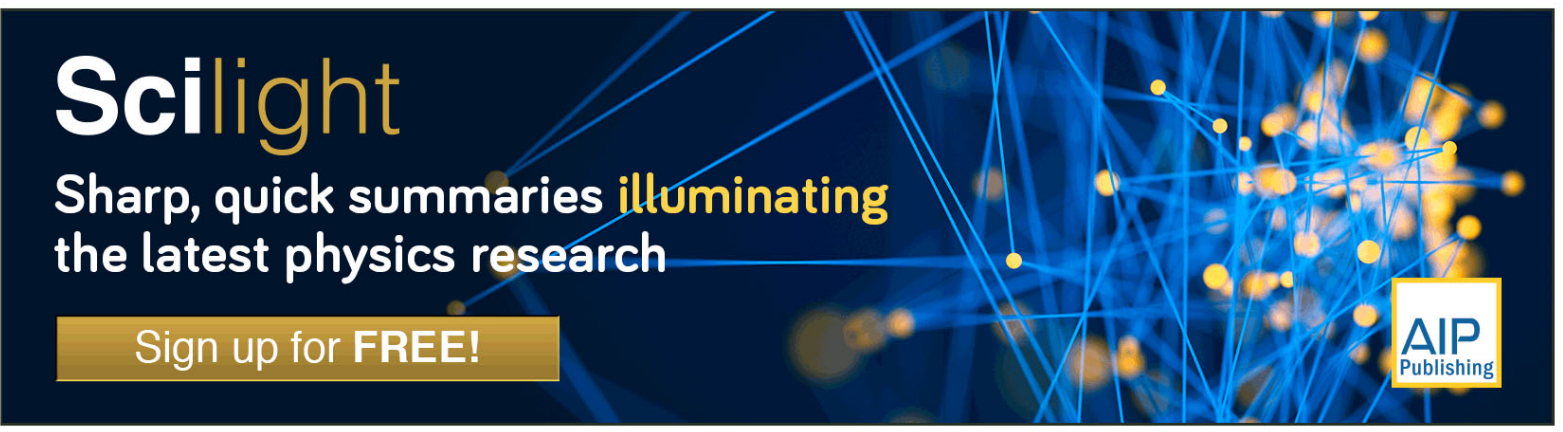




\title{
Landau-Zener-Stückelberg-Majorana interference in a 3D transmon driven by a chirped microwave
}

\author{
Ming Gong, ${ }^{1,2, a)}$ Yu Zhou, ${ }^{3, a)}$ Dong Lan, ${ }^{1}$ Yunyi Fan, ${ }^{3}$ Jiazheng Pan, ${ }^{3}$ Haifeng Yu, ${ }^{1,4, b)}$ \\ Jian Chen, ${ }^{3}$ Guozhu Sun, ${ }^{3,4, c)}$ Yang Yu, ${ }^{1,4}$ Siyuan Han, ${ }^{2}$ and Peiheng Wu ${ }^{3,4}$ \\ ${ }^{1}$ National Laboratory of Solid State Microstructures, School of Physics, Nanjing University, Nanjing 210093, \\ China \\ ${ }^{2}$ Department of Physics and Astronomy, University of Kansas, Lawrence, Kansas 66045, USA \\ ${ }^{3}$ Research Institute of Superconductor Electronics, School of Electronic Science and Engineering, \\ Nanjing University, Nanjing 210093, China \\ ${ }^{4}$ Synergetic Innovation Center of Quantum Information and Quantum Physics, University of Science and \\ Technology of China, Hefei, Anhui 230026, China
}

(Received 24 December 2015; accepted 3 March 2016; published online 15 March 2016; corrected 4 April 2016)

By driving a 3D transmon with microwave fields, we generate an effective avoided energy-level crossing. Then we chirp microwave frequency, which is equivalent to driving the system through the avoided energy-level crossing by sweeping the avoided crossing. A double-passage chirp produces Landau-Zener-Stückelberg-Majorana (LZSM) interference that agree well with the numerical results, especially with the initial state being an eigen-energy state in the center of an avoided level crossing. A time-resolved state tomography measurement is performed in the evolution of LZSM interference, showing an experimental evidence for the dynamical evolution of quantum state. Our method is fully applicable to other quantum systems that contain no intrinsic avoided level crossing, providing an alternative approach for quantum control and quantum simulation. (C) 2016 AIP Publishing LLC. [http://dx.doi.org/10.1063/1.4944327]

Landau-Zener-Stückelberg-Majorana (LZSM) interference $^{1-5}$ is a well known quantum phenomenon, resulting from sweeping a system back and forth across an avoided energy-level crossing in the energy diagram. It has been extensively explored in a lot of systems ${ }^{6}$ including atomic systems, ${ }^{7}$ quantum dots, ${ }^{8}$ and superconducting qubits. ${ }^{9-13}$ In the recent practice of quantum information processing, LZSM interference provides a useful tool to calibrate some crucial characteristics of a system, e.g., the energy-level structure, the coupling strength of the quantum states, and the decoherence time. ${ }^{9,10,14}$ It also finds applications in manipulating quantum states. Conventionally, in order to generate LZSM interference one has to locate an avoided energy-level crossing in the energy diagram of the system. Then one sweeps the external parameter to drive the system across the avoided energy-level crossing, where LandauZener (LZ) transition occurs. The split states of the system evolve along two different paths, accumulating phase difference. When one sweeps the system back and passes the avoided energy-level crossing again, the split states will interfere, creating LZSM interference patterns. However, for some quantum systems, there is no avoided energy-level crossing in the energy diagram. Even worse, their energylevel spacings may be independent of the external bias parameters therefore one cannot drive the system by sweeping the external parameters. A typical case with these two properties is a 3D transmon, ${ }^{15,16}$ which is an improved version of a superconducting qubit. ${ }^{17}$ Although significant amounts of

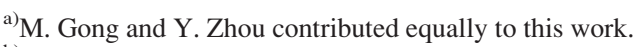

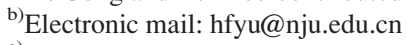

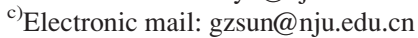

quantum phenomenon have been demonstrated, ${ }^{18-21}$ LZSM interference has not been reported in 3D transmon so far.

Chirping field is a widely used method for coherent population transfer in atomic and molecular systems. ${ }^{22-25}$ In this letter, we realize LZSM interference in a 3D transmon by using chirped microwave. In the rotating frame, a microwave driven 3D transmon exists an effective avoided energy-level crossing. ${ }^{26}$ By gradually chirping the microwave frequency, we can drive the system through the avoided energy-level crossing instead of sweeping the external bias parameter. ${ }^{27}$ By sweeping the system twice across the avoided energylevel crossing, we observe LZSM interference and show the quantum dynamic evolution of LZSM interference changing with the initial states and detuning. The numerical simulated results agree with the experimental data very well.

The sample we used is a transmon qubit centered in a 3D rectangular aluminum (Al 6061-T6 alloy) cavity. The fundamental resonant frequency of the bare cavity is $9.0131 \mathrm{GHz}$. The transmon is fabricated with standard double-angle shadow evaporation of aluminum on a highresistivity silicon substrate. The sample is mounted on the mixing chamber of a dilution refrigerator with base temperature about $20 \mathrm{mK}$. A $\mu$-metal can is used to shield the external magnetic field. In order to achieve high signal-to-noise ratio (SNR), the input signal is heavily attenuated and the low-noise microwave amplifiers have been used for the output signal. ${ }^{16}$ The qubit state is readout by a standard "bright state" readout technique of heterodyne technique. ${ }^{28}$

The truncated Hamiltonian of the two lowest levels $|0\rangle$ and $|1\rangle$ of a 3D transmon qubit are $H_{0}=\hbar \omega_{01}$, where $\omega_{01}$ is the energy difference between $|0\rangle$ and $|1\rangle$. From spectroscopy measurement, we obtain that $\omega_{01} / 2 \pi=5.7744 \mathrm{GHz}$, as shown in Fig. 1(a). With the second excited energy level 
(a)

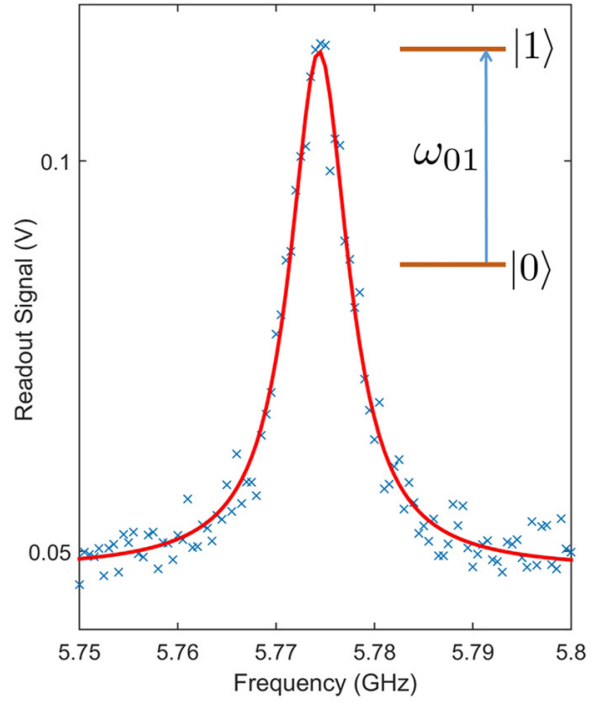

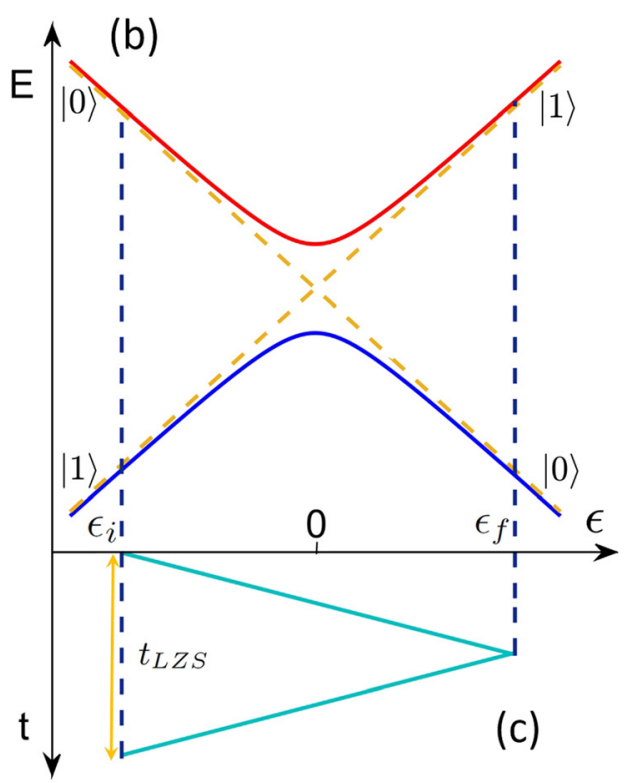

FIG. 1. (a) The spectroscopy of the qubit, with the resonant qubit frequency $\omega_{01} / 2 \pi=5.7744 \mathrm{GHz}$. The inset is a diagram of the energy levels of a 3D transmon qubit. (b) Schematic diagram of the energy-level of a 3D transmon qubit driven by a chirped microwave. (c) Time profile of $\epsilon$ in performing LZSM interference. determined as $\omega_{02} / 2 \pi=11.2744 \mathrm{GHz}$, we calculated the Josephson coupling energy as $E_{J} / h=16.7 \pm 0.1 \mathrm{GHz}$, and the charge energy as $E_{C} / h=274 \pm 2 \mathrm{MHz}^{29}$

If we drive the qubit with microwave $A_{r} \sin \omega_{r} t$, the Hamiltonian of the driven system is identical to a quantum two-level system $(\hbar \equiv 1)$

$$
H=-\frac{1}{2}\left(\epsilon \sigma_{z}+\Omega_{0} \sigma_{x}\right),
$$

where $\epsilon=\omega_{r}-\omega_{01}$ is the detuning and $\Omega_{0}$ is the gap size of the avoided energy-level crossing, as shown in Fig. 1(b), which is proportional to the amplitude of the microwave field $A_{r}$. In general, for a 3D transmon, $\omega_{01}$ is constant. We cannot change $\omega_{01}$ to generate ordinary LZ transition by sweeping the external field. However, in order to sweep the system across the avoided energy-level crossing, we can chirp the microwave frequency $\omega_{r}$ from $\omega_{i}$ to $\omega_{f}$ linearly to cross $\omega_{01}$, i.e., $\epsilon(t)=\omega_{r}(t)-\omega_{01}$. In our experiments, the chirp operation is easily realized by applying intermediate frequency (IF) modulation signals generated by an arbitrary waveform generator (Tektronix 70002) to the I/Q ports of a vector signal generator (R\&S SGS100A). Suppose the local microwave signal is $A_{r} \sin \left(\omega_{01} t\right)$, the waveforms applied on the I and Q ports are quadrature signals, i.e., $\cos \left(\delta_{\omega} t+\phi_{0}\right)$ and $\sin \left(\delta_{\omega} t+\phi_{0}\right)$, respectively, then the modulated microwave waveform is $A_{r} \sin \left(\left(\omega_{01}+\delta_{\omega}\right) t+\phi_{0}\right)$, where $\delta_{\omega}=\epsilon=v t, v$ is the sweeping speed, and $\phi_{0}$ is the initial phase of the modulation. In our experiment, we set $\phi_{0}=0$ so that the coupling between the chirped microwave field and the qubit is in $x$ direction. A chirp operation with same speed is performed to sweep the system back across the avoided level crossing, as shown in Fig. 1(c). The double passage passing the avoided crossing leads to LZSM interference, which is analogous to MachZehnder interferometry in optics. ${ }^{9}$ After the chirp operation, a state tomography measurement ${ }^{30-32}$ is performed to obtain the expectation value of $\left\langle\sigma_{x, y, z}\right\rangle$ of the qubit state.

We investigate LZSM interference for various initial states and detuning. First of all, we choose the gap size of the avoided crossing as $\Omega_{0} / 2 \pi=20 \mathrm{MHz}$ and initialize the state in $|0\rangle$ at $\epsilon_{i} / 2 \pi=-400 \mathrm{MHz}$, satisfying $\left|\epsilon_{i}\right| / \Omega_{0}=20$ $\gg 1$, which indicates that the sweeping starts far away from the center of avoided crossing. Then we can adjust the sweeping range $\epsilon_{f}$ and sweeping time $t_{L Z}$, which are defined by the final frequency of chirp and the chirp speed, respectively. In order to produce LZSM interference, we let the qubit pass the avoided crossing twice. For simplicity, the sweeping speed keeps unchanged for the two passages. Therefore, the whole time for LZSM interference evolution is $t_{L Z S}=2 t_{L Z}$. Fig. 2(a) shows the typical patterns of LZSM interference, where $t_{L Z}$ varies from $1 \mathrm{~ns}$ to $50 \mathrm{~ns}$, and $\epsilon_{f} / 2 \pi$ varies from $-400 \mathrm{MHz}$ to $400 \mathrm{MHz}$. All three components of the qubit state represented by the expectation values of $\left\langle\sigma_{x, y, z}\right\rangle$ are measured by performing the state tomography measurement after the LZSM interference evolution.

In order to confirm the observation, we compare the results with those of the numerical simulation. The quantum dynamics of system can be described with the master equation of the time evolution of the density matrix $\rho$ considering the effects of dissipation

$$
\dot{\rho}=\frac{1}{i \hbar}[H, \rho]-\Gamma[\rho],
$$

where $H$ is the Hamiltonian of the system given by Eq. (1). $\Gamma[\rho]$ describes the decoherence effect in the evolution phenomenologically, including the relaxation time $T_{1}$ and dephasing time $T_{2}^{*}$. By substituting $T_{1}=2.38 \pm 0.13 \mu$ s determined from energy relaxation measurement and $T_{2}^{*}=2.27 \pm 0.33 \mu$ s determined from Ramsey fringe measurement, we obtain the numerical patterns, as shown in the insets of Fig. 2(a). The agreement between the theoretical and experimental results is excellent, indicating the validity of our chirp method in realization of LZSM interference.

For another case, we prepare the initial state in $\frac{1}{\sqrt{2}}(|0\rangle+|1\rangle)$, an energy eigenstate of the system, by applying a resonant $-\pi / 2$ rotation around the $y$ axis. This time we start the evolution from the center of the avoided crossing, i.e., $\epsilon_{i} / 2 \pi=0$. Similar to the previous experiment, we choose $\Omega_{0} / 2 \pi=20 \mathrm{MHz}, \epsilon_{f} / 2 \pi$ ranges from 0 to 

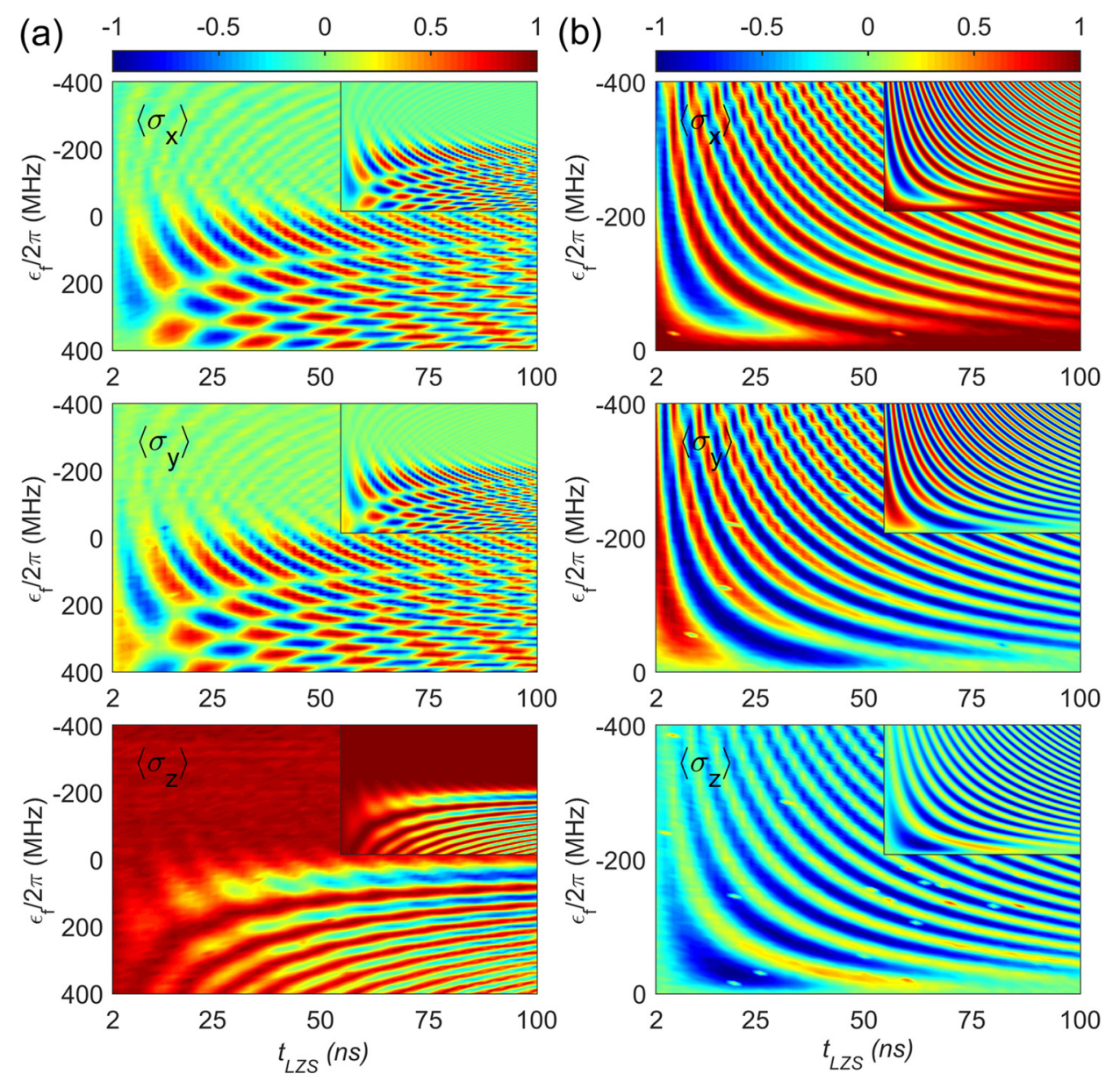

FIG. 2. (a) and (b) Measured expectation values $\left\langle\sigma_{x, y, z}\right\rangle$ of the qubit state as a function of $\epsilon_{f} / 2 \pi$ and $t_{L Z S}$. The insets are the numerical simulations. In (a), the initial state is far away from the avoided energy-level crossing, prepared in $|0\rangle$ at $\epsilon_{i} / 2 \pi=-400 \mathrm{MHz}$. In (b), the initial state is in the center of the avoided energy-level crossing, prepared in $\frac{1}{\sqrt{2}}(|0\rangle+|1\rangle)$ at $\epsilon_{i} / 2 \pi=0$. Almost identical LZSM interference patterns are observed in the experimental and numerical results.

$-400 \mathrm{MHz}$, and $t_{L Z}$ varies from $1 \mathrm{~ns}$ to $50 \mathrm{~ns}$. The results of the expectation values $\left\langle\sigma_{x, y, z}\right\rangle$ of the qubit state are shown in Fig. 2(b), which are also in excellent agreement with the numerical simulations (insets). We would like to mention that LZSM interferences in previous work usually start sweeping the parameter far away from the avoided crossing. Although it is not difficult to theoretically calculate the evolution starting from the center of the avoided crossing with the initial state being the energy eigenstate, there are two obstacles for the experimental investigation. One is how to define the exact center of the avoided crossing. The other is how to create the high fidelity initial eigenstate which is the superposition of diabatic states. Our chirp method solves both problems simultaneously: The avoided crossing is easily defined at $\omega_{r}(t)=\omega_{01}$, i.e., $\epsilon=0$. At the same time, the initial state can be prepared with on-resonant microwave; therefore, one can initialize the state to any point on Bloch sphere, including the energy eigenstate of the system.

The excellent agreement between the experimental data and the simulation results indicates that with a chirp technique one can realize and investigate LZSM interference completely in a system without intrinsic avoided energylevel crossings. The splitting of the avoided crossing and the sweeping range can be tuned conveniently by changing the chirp parameters such as $\Omega_{0}$ and $\delta_{\omega}$. We are able to prepare the initial state completely in one of the eigenstates without any leakage to the other one.

Using the method of chirping frequency, one can also perform time-resolved state tomography measurement to obtain the dynamical evolution of the qubit during LZSM interference. We set $\epsilon_{i} / 2 \pi=-400 \mathrm{MHz}$ and $\epsilon_{f} / 2 \pi=400 \mathrm{MHz}$. The total evolution time is $40 \mathrm{~ns}$. At each step which is $1 \mathrm{~ns}$, the state of the qubit is measured. Then the density matrix in the dynamical evolution of the qubit in LZSM interference can be obtained. For example, the density matrix of the qubit at evolution time $\mathrm{T}=1 \mathrm{~ns}, 24 \mathrm{~ns}$, and $40 \mathrm{~ns}$ is shown in Fig. 3(a), representing the qubit state at the beginning, after the first LZ transition, and at the end of an LZSM interference process, respectively. The state evolution confirms the physics picture of LZSM interference, in which the system starts from the diabatic state, separates to a superposition state after the first LZ transition, then interferes at the second LZ transition. Similarly, we also investigate the evolution starting from the center of avoided crossing, i.e., $\epsilon_{i} / 2 \pi=0$. Here $\epsilon_{f} / 2 \pi=-200 \mathrm{MHz}$ and $t_{L Z S}=160 \mathrm{~ns}$. In this situation, the system starts from the superposition state and interferes when it passes the avoided crossing. We perform the state tomography measurement in the process to obtain the density matrix of the qubit at $\mathrm{T}=1 \mathrm{~ns}, 84 \mathrm{~ns}$, and 160ns shown in Fig. 3(b), representing the qubit state at the beginning, middle, and end of the evolution. The evolution of the density matrix clearly shows the process mentioned above. Numerical simulations for both cases agree well with the measured evolution of density matrix.

Compared with the conventional method of realizing LZSM interference by sweeping external bias parameter, our chirp method has several advantages. The first one is that during the evolution, all parameters of the qubit, such as $T_{1}$, 
(a)
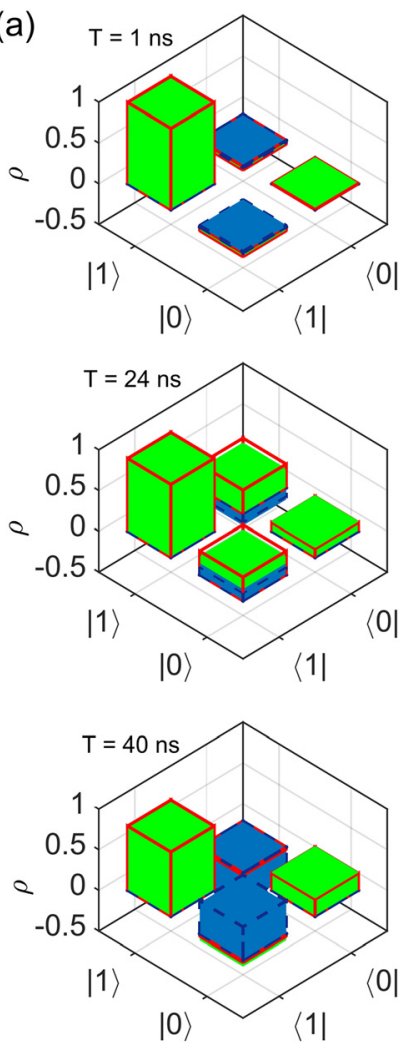

(b)
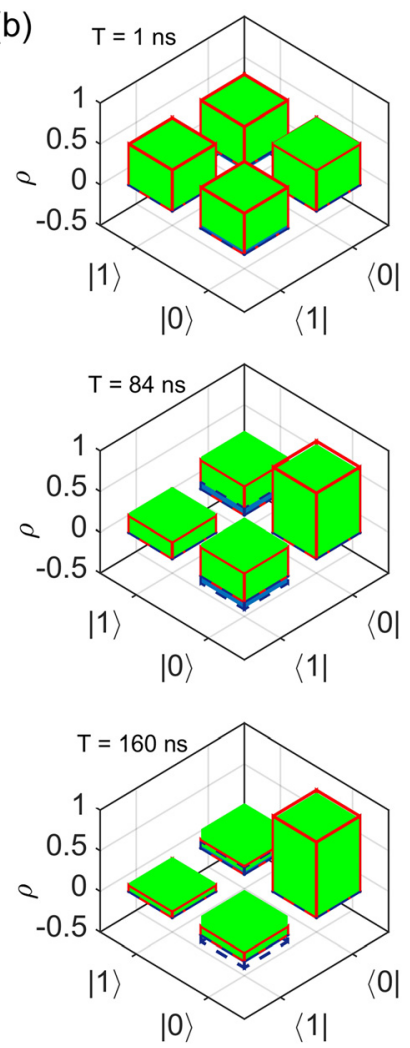

FIG. 3. (a) and (b) State tomography of the qubit in the evolution of LZSM interference. The real (imaginary) parts of the experimental and numerical simulated density matrix are shown as green (blue) solid bars and red solid (blue dashed) lines, respectively. In (a), the initial state is prepared in $|0\rangle$ at $\epsilon_{i} / 2 \pi=-400 \mathrm{MHz}$. The total evolution time is $40 \mathrm{~ns}$ and $\epsilon_{f} / 2 \pi$ is $400 \mathrm{MHz}$. The density matrix of the qubit at $\mathrm{T}=1 \mathrm{~ns}, 24 \mathrm{~ns}$, and $40 \mathrm{~ns}$ are presented, respectively. In (b), the initial state is prepared in $\frac{1}{\sqrt{2}}(|0\rangle+|1\rangle)$ at $\epsilon_{i} / 2 \pi=0$. The total evolution time is $160 \mathrm{~ns}$ and $\epsilon_{f} / 2 \pi$ is $-200 \mathrm{MHz}$. The density matrix of the qubit at $\mathrm{T}=1 \mathrm{~ns}, 84 \mathrm{~ns}$, and $160 \mathrm{~ns}$ are presented, respectively. (Multimedia view) [URL: http://dx.doi.org/10.10.1063/ 1.4944327.1] [URL: http://dx.doi.org/10.10.1063/1.4944327.2]

$T_{2}^{*}$, and the coupling strength between the qubit and the external driving field keep unchanged in the whole process. These parameters usually depend on the external bias. Therefore, when we generate LZSM interference by sweeping external bias, the evolution may be complicated. Second, the chirp range $\epsilon_{i}$ and $\epsilon_{f}$ in our method is not limited by the structure of the qubit energy diagram. For instance, it is not affected by the nearby intrinsic avoided energy-level crossings or the additional splittings caused by the coupling to the microscopic two-level systems. Third, it is easy to control the chirp velocity and the coupling strength between the driving field and qubit by controlling the microwave frequency and power.

In summary, we realize LZSM interference in a superconducting 3D transmon which has constant energy level spacing thus containing no intrinsic avoided energy-level crossing. An effective avoided crossing is created by the microwave field. Then we chirp the microwave frequency and drive the system through the avoided crossing. By sweeping the system twice across the avoided crossing, we observe LZSM interference. Our method can be applied to the systems whose energy diagrams lack intrinsic avoided energy-level crossings and/or cannot be changed rapidly by sweeping external parameters. As long as they can interact with external microwave

irradiation, one can generate LZSM interference to calibrate some crucial characteristics of the system and to conduct quantum control and/or quantum simulation.

This work was partially supported by the SKPBR of China (2011CB922104), NSFC (11474154, 93121310, 11274156, 61521001, BK2012013, 11474152), PAPD, a doctoral program (20120091110030), and Dengfeng Project B of Nanjing University. S.H. was supported in part by NSF (PHY-1314861).

${ }^{1}$ L. Landau, Phys. Z. Sowjetunion 1, 88 (1932).

${ }^{2}$ L. Landau, Phys. Z. Sowjetunion 2, 46 (1932).

${ }^{3}$ C. Zener, Proc. R. Soc. London, Ser. A 137, 696 (1932).

${ }^{4}$ E. Stückelberg, Helv. Phys. Acta 5, 369 (1932).

${ }^{5}$ F. Di Giacomo and E. E. Nikitin, Phys.-Usp. 48, 515 (2005).

${ }^{6}$ S. N. Shevchenko, S. Ashhab, and F. Nori, Phys. Rep. 492, 1 (2010).

${ }^{7}$ C. S. E. van Ditzhuijzen, A. Tauschinsky, and H. B. van Linden van den Heuvell, Phys. Rev. A 80, 063407 (2009).

${ }^{8}$ H. Ribeiro and G. Burkard, Phys. Rev. Lett. 102, 216802 (2009).

${ }^{9}$ W. D. Oliver, Y. Yu, J. C. Lee, K. K. Berggren, L. S. Levitov, and T. P. Orlando, Science 310, 1653 (2005).

${ }^{10}$ M. Sillanpää, T. Lehtinen, A. Paila, Y. Makhlin, and P. Hakonen, Phys. Rev. Lett. 96, 187002 (2006).

${ }^{11}$ A. Izmalkov, S. H. W. van der Ploeg, S. N. Shevchenko, M. Grajcar, E. Ilichev, U. Hbner, A. N. Omelyanchouk, and H. G. Meyer, Phys. Rev. Lett. 101, 017003 (2008).

${ }^{12}$ G. Sun, X. Wen, Y. Wang, S. Cong, J. Chen, L. Kang, W. Xu, Y. Yu, S. Han, and P. Wu, Appl. Phys. Lett. 94, 102502 (2009).

${ }^{13}$ G. Sun, X. Wen, B. Mao, J. Chen, Y. Yu, P. Wu, and S. Han, Nat. Commun. 1, 51 (2010).

${ }^{14}$ A. V. Shytov, D. A. Ivanov, and M. V. Feigel'man, Eur. Phys. J. B 36, 263 (2003).

${ }^{15}$ H. Paik, D. I. Schuster, L. S. Bishop, G. Kirchmair, G. Catelani, A. P. Sears, B. R. Johnson, M. J. Reagor, L. Frunzio, L. I. Glazman et al., Phys. Rev. Lett. 107, 240501 (2011).

${ }^{16}$ C. Rigetti, J. M. Gambetta, S. Poletto, B. L. T. Plourde, J. M. Chow, A. D. Corcoles, J. A. Smolin, S. T. Merkel, J. R. Rozen, G. A. Keefe et al., Phys. Rev. B 86, 100506 (2012)

${ }^{17}$ J. Clarke and F. K. Wilhelm, Nature 453, 1031 (2008).

${ }^{18}$ M. H. Devoret and R. J. Schoelkopf, Science 339, 1169 (2013).

${ }^{19}$ P. Campagne-Ibarcq, E. Flurin, N. Roch, D. Darson, P. Morfin, M. Mirrahimi, M. H. Devoret, F. Mallet, and B. Huard, Phys. Rev. X 3, 021008 (2013).

${ }^{20}$ G. Kirchmair, B. Vlastakis, Z. Leghtas, S. E. Nigg, H. Paik, E. Ginossar, M. Mirrahimi, L. Frunzio, S. M. Girvin, and R. J. Schoelkopf, Nature 495, 205 (2013).

${ }^{21}$ K. Murch, S. Weber, C. Macklin, and I. Siddiqi, Nature 502, 211 (2013).

${ }^{22}$ B. Broers, H. van den Heuvel, and L. Noordam, Phys. Rev. Lett. 69, 2062 (1992).

${ }^{23}$ S. Chelkowski, A. D. Bandrauk, and P. B. Corkum, Phys. Rev. Lett. 65, 2355 (1990).

${ }^{24}$ J. Melinger, S. R. Gandhi, A. Hariharan, D. Goswami, and W. Warren, J. Chem. Phys. 101, 6439 (1994).

${ }^{25}$ J. Cao, C. J. Bardeen, and K. R. Wilson, Phys. Rev. Lett. 80, 1406 (1998).

${ }^{26}$ G. Sun, X. Wen, B. Mao, Y. Yu, J. Chen, W. Xu, L. Kang, P. Wu, and S. Han, Phys. Rev. B 83, 180507 (2011).

${ }^{27}$ J. Zhou, P. Huang, Q. Zhang, Z. Wang, T. Tan, X. Xu, F. Shi, X. Rong, S. Ashhab, and J. Du, Phys. Rev. Lett. 112, 010503 (2014).

${ }^{28}$ M. D. Reed, L. DiCarlo, B. R. Johnson, L. Sun, D. I. Schuster, L. Frunzio, and R. J. Schoelkopf, Phys. Rev. Lett. 105, 173601 (2010).

${ }^{29}$ J. Koch, T. M. Yu, J. Gambetta, A. A. Houck, D. I. Schuster, J. Majer, A. Blais, M. H. Devoret, S. M. Girvin, and R. J. Schoelkopf, Phys. Rev. A 76, 042319 (2007).

${ }^{30}$ M. Steffen, M. Ansmann, R. McDermott, N. Katz, R. C. Bialczak, E. Lucero, M. Neeley, E. M. Weig, A. N. Cleland, and J. M. Martinis, Phys. Rev. Lett. 97, 050502 (2006).

${ }^{31}$ P. J. Leek, J. M. Fink, A. Blais, R. Bianchetti, M. Göppl, J. M. Gambetta, D. I. Schuster, L. Frunzio, R. J. Schoelkopf, and A. Wallraff, Science 318, 1889 (2007).

${ }^{32}$ C. Deng, J.-L. Orgiazzi, F. Shen, S. Ashhab, and A. Lupascu, Phys. Rev. Lett. 115, 133601 (2015). 